\title{
Arte en la cárcel y solidaridad internacional. Entrevista a Juan Baladán Gadea
}

\author{
Art in prison and international solidarity. Interview with Juan Baladán Gadea
}

Por Moira Cristiá*

Nacido en Treinta y Tres del Olimar en 1942, Juan Baladán Gadea es un poeta, músico y compositor uruguayo que sufrió la prisión por razones políticas entre 1971 y 1985, tras lo cual se exilió en Breccia (Italia). Durante sus catorce años de reclusión, su actividad artística se tornó en una forma de resistencia frente a la opresión, así como motor de existencia en esas difíciles condiciones de vida. Organismos internacionales tales como Amnesty International, así como de la Association internationale de défense des artistes victimes de la répression dans le monde (AIDA) se movilizaron por su liberación. Fundada en 1979 y radicada en varios países europeos y en Estados Unidos, AIDA tenía como objetivo denunciar a través de acciones creativas la censura y violencia sufridas por artistas en cualquier parte del mundo. Además de defender casos de Europa del Este, Asia o África, AIDA actuó en la esfera pública transnacional para presionar a las dictaduras latinoamericanas, empleando la creación como forma de combatir el autoritarismo en el mundo. Motivada por el estudio de dicha asociación, la entrevista a Juan Baladán Gadea permite reflexionar sobre la maquinaria represiva desplegada en América Latina y los mecanismos culturales de resistencia a la misma.

Palabras clave: Uruguay, solidaridad internacional, artistas, Derechos Humanos, dictaduras latinoamericanas, represión, exilio

Born in Treinta y Tres del Olimar in 1942, Juan Baladán Gadea is an Uruguayan poet, musician and composer who was imprisoned for political reasons from 1971 to 1985 . When he was finally released, he exiled in Breccia (Italy). During his fourteen years in prison, his artistic activity became a form of resistance to oppression and a way of enduring those difficult living conditions. Some international organizations such as Amnesty International and the International

\footnotetext{
* Argentina. Doctora en Historia y Civilizaciones por l'École des Hautes Études en Sciences Sociales (París). Investigadora asistente del Consejo Nacional de Investigaciones Científicas y Técnicas de Argentina (CONICET), en el Instituto de Investigaciones Gino Germani, Universidad de Buenos Aires. Mail: moicristia@gmail.com
} 
Association for the Defense of Artists victims of repression worldwide (AIDA) mobilized for his liberation. Founded in 1979 and functioning in different European countries and the United States, AIDA aimed to denounce through creative actions the censorship and violence suffered by artists all over the world. Besides defending different cases in Eastern Europe, Asia and Africa, AIDA acted in the transnational public sphere to exert pressure on Latin American dictatorships, using the creation as a way to struggle authoritarianism around the world. Motivated by the study of this association, the interview with Juan Baladán Gadea allows reflecting on the repressive machinery deployed in Latin America and the cultural mechanisms of resistance to it.

Keywords: Uruguay, international solidarity, transnational history, artists, Human Rights, Latin American dictatorships, repression, exile

Juan Baladán Gadea, poeta, músico y compositor. Con 29 años de edad, fue encarcelado por razones políticas, encierro que se extendió durante catorce años hasta su liberación y exilio en la ciudad de Breccia (Italia) en $1985^{1}$. Durante ese largo tiempo de reclusión, su actividad artística no sólo no se interrumpió, sino que se tornó en una forma de resistencia frente a la represión, así como motor de existencia en esas difíciles condiciones de vida. En dicho ámbito hostil, además de perfeccionar el estudio de instrumentos musicales (flauta y guitarra), Baladán Gadea profundizó sus conocimientos sobre folklore sudamericano, compuso piezas musicales, escribió y musicalizó poesía, a la vez que, como sus compañeros, se refugió en la lectura o en la elaboración de artesanías. Aún durante su encierro, una obra musical de su autoría fue estrenada en Europa: "Sierras del Yerbal" sonó por primera vez en Róterdam en mayo de 1983 por un grupo de músicos holandeses. Tras su liberación, algunos de los materiales creados durante su reclusión encontraron forma pública, como fue el disco Canzoni d'amore e di speranza (Juan Baladán Gadea, Brescia, 1986) o su primer libro Voy soñando calles (Milano, Edizioni Mondo Nuovo, 1989). Instalado con su familia en Europa hasta la actualidad, Juan Baladán Gadea continúa su carrera de compositor y poeta ${ }^{2}$.

Aunque sin éxito concreto, el apoyo solidario de artistas y organizaciones internacionales tales como Amnesty International y AIDA (Association Internationale de Défense des Artistes victimes de la répression dans le monde) fue significativo. Esta última, fundada en 1979 tras una visita de Ariane Mnouchkine y Claude Lelouch de Santiago de Chile, Buenos Aires y Montevideo para evaluar las condiciones de los artistas en dichos

\footnotetext{
${ }^{1}$ Sobre la diversidad del exilio uruguayo según la multiplicidad de experiencias, pertenencia social y política, tipo de militancia y país de refugio, consultar: Silvia Dutrénit Bielous (cood.) El Uruguay del exilio: gente, circunstancias, escenarios, Montevideo, Trilce, 2006.

${ }^{2}$ Para una síntesis biográfica más completa, ver la redactada por Alfredo Alzugarat en el blog "Al otro lado del río. Una lata con tierra de Treintaytres, labrada y sembrada... al otro lado del rio", URL: http://blogs.montevideo.com.uy/blognoticia_18190_1.html
} 
países bajo dictaduras militares, se expandió rápidamente por fuera de Francia: Alemania, Bélgica, Suiza, Holanda y Estados Unidos. Fue su sección holandesa la que actuó rápidamente por Baladán Gadea: concibió algunas postales para reclamar por su liberación, envió cartas al Embajador uruguayo en La Haya y al Embajador holandés en Montevideo, y exhortó a las autoridades de su país de origen a liberarlo alegando que se trataba de un artista de gran valor para Uruguay y para el mundo, por lo que se comprometían a recibirlo en Europa y ofrecerle asistencia financiera el tiempo que fuera necesario ${ }^{3}$.

La asociación también impulsó una petición que fue dirigida al gobierno de Uruguay, reuniendo las firmas de artistas holandeses de renombre como Kess Stolwijk, Wim Crouwel, Mensje van Keulen, Jan Siebelink, Ritsaert ten Cate, Ton Koopman and Kees Van Kooten, el director italiano Pier Luigi Alli, el dramaturgo y director de teatro inglés Pip Simmons, el compositor y pianista Ivar Mikhashoff, el cantante Joan la Barbara, el director de orquesta Michael Tilson Thomas (estos últimos estadounidenses) y la coreógrafa alemana Pina Bausch. En particular, fue primero el pianista argentino Miguel Ángel Estrella -quien había compartido reclusión con Juan Baladán Gadea- y luego el violinista Yehudi Menuhin quienes dieron a conocer este caso e intentaron llamar la atención de las autoridades ${ }^{4}$. En junio de 1983, en la reunión internacional anual de AIDA, tras un largo debate, se decidió suspender las acciones por este caso ya que temían que las acusaciones de su pertenencia a Tupamaros pudieran servir de argumentos para deslegitimar a la asociación. Sin embargo, no descartaron que sus miembros siguieran impulsando este caso de manera personal o creando un comité de solidaridad ad hoc ${ }^{5}$.

\section{$*$}

La siguiente entrevista se realizó en el marco de una investigación más amplia sobre AIDA (Asociación Internacional de Defensa de Artistas víctimas de la represión en el mundo), y en particular en el apoyo de esta asociación a artistas latinoamericanos ${ }^{6}$. Tras el caso más reconocido y para el cual se desplegó una amplia campaña de solidaridad internacional encabezada por Yves Haguenauer bajo la dirección simbólica de Nadine Boulanger y Yehudi Menuhin -el del pianista argentino preso por la dictadura uruguaya Miguel Ángel Estrella ${ }^{7}$ - las acciones que AIDA impulsó por el caso de Juan Baladán Gadea, así como por

\footnotetext{
3 AIDA "Public appeal to the government of Uruguay", firmado por Aad Hoetjes, presidente de AIDAHolanda, 17 de junio de 1982. Archivos AIDA Francia, La Cartoucherie, París.

${ }^{4}$ AIDA Press release, Amsterdam, 17 de junio de 1982. Archivos AIDA Francia, La Cartoucherie, París.

${ }^{5}$ Actas de la reunión internacional en la Cartoucherie, París, 18 de junio de 1983. Archivos AIDA Francia, La Cartoucherie, París.

${ }^{6}$ Ver Moira Cristiá, "Imaginación y resistencia antidictatorial en los años ochenta. La acción por América Latina de la Asociación Internacional de Defensa de Artistas Víctimas de la Represión en el Mundo (AIDA)”, Revista Izquierdas,36, noviembre 2017.

${ }^{7}$ Sobre este caso en particular consultar: Esteban Buch y Anaïs Fléchet "La musique en prison. La campagne pour la libération de Miguel Ángel Estrella (1977-1980) », Annales. Histoire, Sciences Sociales, 2017 (en prensa). Los archivos de Yves Haguenauer sobre el comité de solidaridad Miguel Ángel Estrella están disponibles en la biblioteca del Institut d'Histoire de Temps Présent [ARC 3018 (1-9)].
} 
el de la pianista uruguaya Alba González Souza, motivaron el intento de escuchar esas voces. Las referencias en los trabajos de Alfredo Alzúgarat, quien fuera también compañero de reclusión de Baladán Gadea, nos condujeron a contactar al escritor y crítico uruguayo, quien generosamente funcionó de vínculo con el músico y poeta exiliado en Breccia ${ }^{8}$. Esta entrevista se realizó de manera escrita, por lo que el cuestionario fue enviado el 06/05/2017 y el entrevistado retornó sus respuestas el 07/06/2017.

M. C. Según tengo entendido, Ud. estuvo preso de 1971 a 1985 y se lo acusaba de "atentado a la constitución, en el grado de conspiración", "asociación para delinquir", "rapiña”, es decir que lo vinculaban con el MLN Tupamaros. Según los informes de AIDA, ud. representaba al magisterio y participaba del CNT. ¿Qué tipo de militancia llevaba a cabo por ese entonces?

J. B. G. Yo fui detenido el 17/10/1971 y liberado, por la Ley de Amnistía votada por el nuevo parlamento democrático, el 10/03/1985. Mi militancia era absolutamente legal dentro del marco del Frente Amplio -nacido ese mismo añoen la Lista 99 liderada por el Senador Zelmar Michelini y en el Sindicato. No fui dirigente de ninguna organización política ni sindical, era un adherente como tantos. Era conocido en mi ciudad y en otros ambientes culturales por mi actividad musical.

M. C. Alfredo Alzugart en su libro "Trincheras de papel"9 menciona que el Penal Libertad fue renovado en 1972 y que Walter Phillipps-Treby fue uno de los primeros presos, con el nro. 010. De manera que ud. fue un preso político "temprano”, ¿verdad? ¿Tiene idea a qué se debió? ¿Estuvo durante todo el resto del período en el Penal Libertad?

J. B. G. Después de mi detención y el subsiguiente "tratamiento militar" fui conducido al Penal de Punta Carretas al entonces llamado "Tercer Piso Especial". Allí estaban detenidos José (Pepe) Mujica Cordano, Jorge Zabalza, otros dirigentes del MLN, Walter Phillipps-Treby y muchos compañeros más. Algunos éramos nuevos detenidos y otros habían sido "recapturados", después de la fuga masiva del 06/09/71 de 106 prisioneros políticos, a través de un túnel construído desde el interior del Penal. El 12/04/1972 se produjo la fuga de 15 prisioneros políticos a través de un túnel (esta vez construído desde el exterior) entre los que se contaban José (Pepe) Mujica Cordano, Jorge Zabalza y otros dirigentes del MLN allí detenidos. Después de esta fuga los militares procedieron al traslado de un grupo de prisioneros, entre ellos Walter Phillipps-Treby, a la Cárcel de Punta de Rieles.

\footnotetext{
${ }^{8}$ Agradezco la ayuda de Germán Silveira y de Julio Osaba para contactar a Alfredo Alzúgarat, y a éste último por ponerme en contacto con Juan Baladán Gadea.

${ }^{9}$ Alfredo Alzugarat, Trincheras de papel. Dictadura y literatura carcelaria en Uruguay, Montevideo, Trilce, 2007.
} 
Meses más tarde, cuando quedó habilitado el Penal de Libertad, este fue el primer grupo trasladado allí. Después siguió el traslado de prisioneros desde los diferentes cuarteles del Ejército de todo el país y de tanto en tanto tocaba a algún pequeño grupo desde Punta Carretas. Yo fui trasladado al Penal de Libertad en los días siguientes a la Navidad de 1974 (no recuerdo la fecha exacta) y me asignaron el $\mathrm{N}^{\circ}$ 1674. Allí estuve hasta mi liberación.

M. C. En 1982, le extendieron la pena que le habían atribuido en 1976 por 12 años por haber participado en una huelga de hambre por la desaparición de compañeros. ¿Cómo se desarrolló esta acción de resistencia?

J. B. G. Las condiciones de vida en el Penal de Libertad son bastantes conocidas hoy porque ha habido una importante actividad de difusión hacia la opinión pública que abarca desde trabajos realizados en el ambiente de la Universidad de la República hasta una infinidad de libros escritos por los propios ex prisioneros políticos. En pocas palabras podría definir aquella experiencia, como la demencial puesta en práctica de un sistema carcelario que reducía la libertad de los prisioneros al mínimo vital, que creaba una situación de profunda ansia y estrés continuado, aplicando una sistemática y sutil tortura psicológica que se prolongó por muchos años, con la finalidad explícita de destruir política y psicológicamente a todos los que allí habíamos sido llevados. Cuando un compañero me comunicó que en una determinada fecha se iniciaría una huelga de hambre como acción de protesta, decidí participar. Nos habían quitado todo; pero aún nos quedaba la dignidad. Sabía que esa acción no tendría éxito, pero era necesaria. Apenas comenzó el ayuno fuimos puestos en aislamiento y cuando al final cada uno volvió a su celda me enteré de la violenta reacción de los militares que se llevaron a algunos compañeros a los cuarteles y los torturaron. Uno de ellos, Hugo Dermit, murió a causa de la tortura. Algunos meses después me llevaron al Tribunal Militar, en Montevideo y un funcionario me leyó un documento firmado por el fiscal donde me acusaba de algunos delitos que, diciendo la verdad, me provocaron gran confusión porque era imposible que yo los hubiera cometido, para eso era necesario vivir libre en alguna ciudad.

M. C. ¿Qué vínculo tuvo con Miguel Ángel Estrella? ¿Sabe si fue él quien propuso su caso a AIDA? ¿Por qué fue la sección holandesa de la organización internacional la que impulsó las acciones por su caso en 1982 mientras que Miguel Ángel se encontraba en Francia desde 1980?

J. B. G. Comienzo esta respuesta con algo que no está en la pregunta. Cuando Miguel Ángel Estrella fue trasladado al Penal de Libertad más de la mitad de los prisioneros estábamos viviendo entre el sexto y octavo año de reclusión y el peso de aquel sistema se sentía; pero de eso no se hablaba casi nunca. Cuando llegaba un nuevo compañero veíamos una persona enflaquecida, psicológicamente abatida, a 
veces con visibles señales físicas de la tortura apenas sufrida. Pocos, muy pocos recuerdan como estaba fisicamente Miguel Ángel cuando entró al Celdario del Penal, porque sólo vimos su sonrisa grande y clara que transmitía optimismo. Su sonrisa nos cautivó y nos desorientó, la vida chiquita de la celda ya nos había marcado sin que lo percibiéramos, pero al mismo tiempo aquella sonrisa nos reavivó la esperanza. Poco después que Miguel Ángel llegara al Piso 4, me llegó una cartita suya a través de un correo clandestino (yo estaba en el Piso 5), comenzó así una amistad que ha tenido gran importancia en mi vida. Después aprovechamos de un momento de "afloje", debido a que los militares que se preparaban a poner en práctica un referendum que, de triunfar, les permitiría perpetuarse en el poder, aplicaban las reglas con menos rigor y hasta permitieron la entrada en el Penal a la Cruz Roja Internacional. Fue así que Miguel Ángel y yo nos inscribimos en un pequeño grupo de prisioneros que después de cena iba a lavar la cocina, que era enorme, a pesar de que estábamos en Pisos diferentes. Mientras cumplíamos con nuestra tarea Miguel Ángel me daba lecciones de música que incluían noticias sobre los estilos de composición más importantes en ese momento. Me dio consejos que me ayudaron en mi búsqueda de un estilo de composición que une el folklore sudamericano con la experiencia clásica y también, esto es extraordinario, me dio lecciones de piano sin piano que me esclarecieron las ideas acerca de problemas técnicos relacionados con la composición. Fue Miguel Ángel que propuso mi caso a AIDA y no tengo ninguna información del porqué se ocupó de esto la sección holandesa.

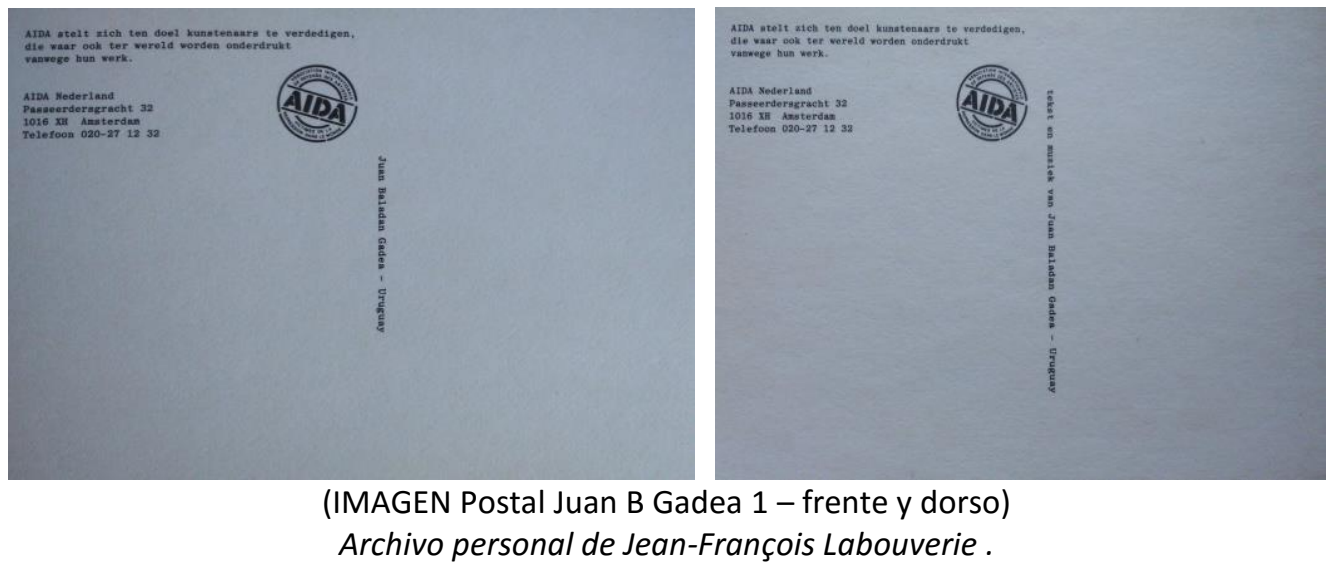

M. C. ¿Y con Yehudi Menuhin, quien también fue un impulsor de su causa? ¿Lo conoció en alguna ocasión?

J. B. G. Cuando AIDA comenzó a ocuparse de mi situación yo no conocía a Yehudi Menuhin personalmente sólo era un admirador de sus interpretaciones, en especial de su versión del Concierto para Violín y Orquesta de Beethoven, y del Concierto para Violín y Orquesta $\mathrm{N}^{\circ} 2$ de Paganini. Algunos meses después de mi 
liberación, en noviembre de 1985, el Maestro Menuhin dio un Concierto aquí en Brescia. Pude hablar con él durante el intervalo después de la primera parte. No sabía de mi liberación. Me estrechó la mano y me dijo: "Estoy convencido que ahora Ud. [es] verdaderamente libre aquí en Italia, hay lugares [de] los que nadie sale". Otras personas presentes intervinieron en la conversación y al final del encuentro el Maestro agregó: "Hoy todos debemos pensar en la dirección que ha tomado la vida en general, no es una cuestión que podamos dejar de lado, ni tomar posición por fuerza, pero no podemos dejar que éstos (se refería a los dictadores) tomen decisiones por nosotros".

M. C. ¿Tiene idea de qué tipo de campaña llevó AIDA por su caso además del envío de postales y una petición firmada por personalidades reconocidas del mundo de la cultura?

J. B. G. El 3 de mayo de 1983 se realizó un Concierto en Rotterdam por la liberación de los presos políticos de Uruguay organizado por AIDA Holanda. En esa oportunidad fue ejecutada una composición mía para orquesta. La partitura había sido sacada clandestinamente de Libertad por Walter Díaz Marrero en 1980 cuando fue liberado y posteriormente transportada a Europa. En abril de 1987 en el programa radial de AIDA "Literama", transmitido por Radio Hilversum 4, fue leída por un actor una poesía mía y a continuación fue transmitida una canción interpretada por mí. También en la revista LITERAMA AIDA fueron publicadas algunas de mis poesías.

M. C. ¿Recibió correspondencia o visita de alguno de los integrantes de AIDA? En el año 1979 viajó Ariane Mnouchkine pero no pudo acceder a visitar a Miguel Ángel Estrella.

J. B. G. No, no recibí ninguna correspondencia de AIDA. En 1983 (no puedo precisar la fecha) el embajador holandés en Uruguay, por órden de su gobierno, fue al Penal de Libertad con la intención de visitarme, pero no le permitieron verme. Le dijeron que yo estaba bien y le invitaron a entrevistarse con Padre Hugo Bordoli, Párroco de la pequeña ciudad de Libertad cercana al Penal. El Embajador tuvo un encuentro de más de dos horas con Padre Bordoli el cual le puso al corriente de la dura situación en la que nos encontrábamos. Los militares no me dijeron nada de esta visita del Embajador holandés, lo supe unos días más tarde porque me lo comunicó Padre Bordoli cuando fue a visitarme. 


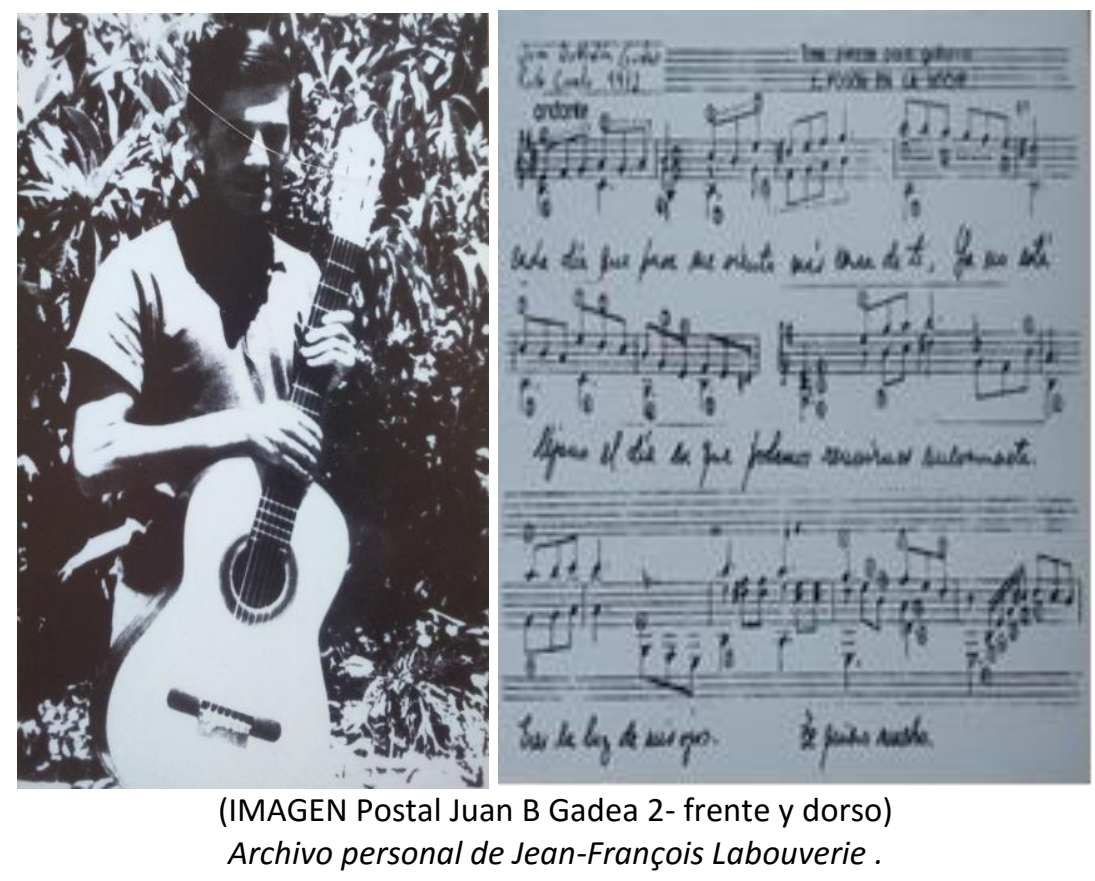

M. C. ¿Le informaron cuando abandonaron su caso o lo derivaron a otra organización?

J. B. G. No, no recibí ninguna información oficial de parte de AIDA. Pero en aquellas condiciones de incomunicación con el exterior de la cárcel en que nos tenían, era imposible darme una comunicación importante como ésta. En la visita de fin de año de 1983, que se realizó en el jardín sin que las guardias controlaran lo que hablábamos, me lo dijo Padre Bordoli. Nos detuvimos a conversar sobre este tema, él estaba muy dolido por lo que había sucedido y yo le dije que comprendía el motivo, sosteniendo al mismo tiempo que era más importante mantener el buen nombre de AIDA para no dar la posibilidad de ataques futuros que de otro modo llegarían. Años más tarde pude leer el contenido del documento de protesta presentado por los militares: al noventa y nueve por ciento decía falsedades y comprendí mejor el disgusto de Padre Bordoli.

M. C. ¿Qué otras organizaciones internacionales se acercaron a su caso además de Amnesty International y AIDA?

J. B. G. En mayo de 1984 la CGIL (Confederazione Generale Italiana del Lavoro) envió a Uruguay al Profesor Marino Ruzzenenti con la misión específica de visitarme y verificar las condiciones en las que yo me encontraba. También a él le fue negada la posibilidad de verme. De esta acción de solidaridad conmigo de parte de la CGIL me enteré cuando llegué a Italia. 
M. C. ¿Conserva Ud., documentos referidos a AIDA?

J. B. G. Tengo algunos documentos (pocos) que conservó mi esposa, y que le fueron entregados en 1983 por activistas de AIDA Holanda con motivo del Concierto de Rotterdam más arriba citado. Además, tengo una fotografía que publicó el Diario Brescia Oggi unos días después de mi breve encuentro con Yehudi Menuhin.

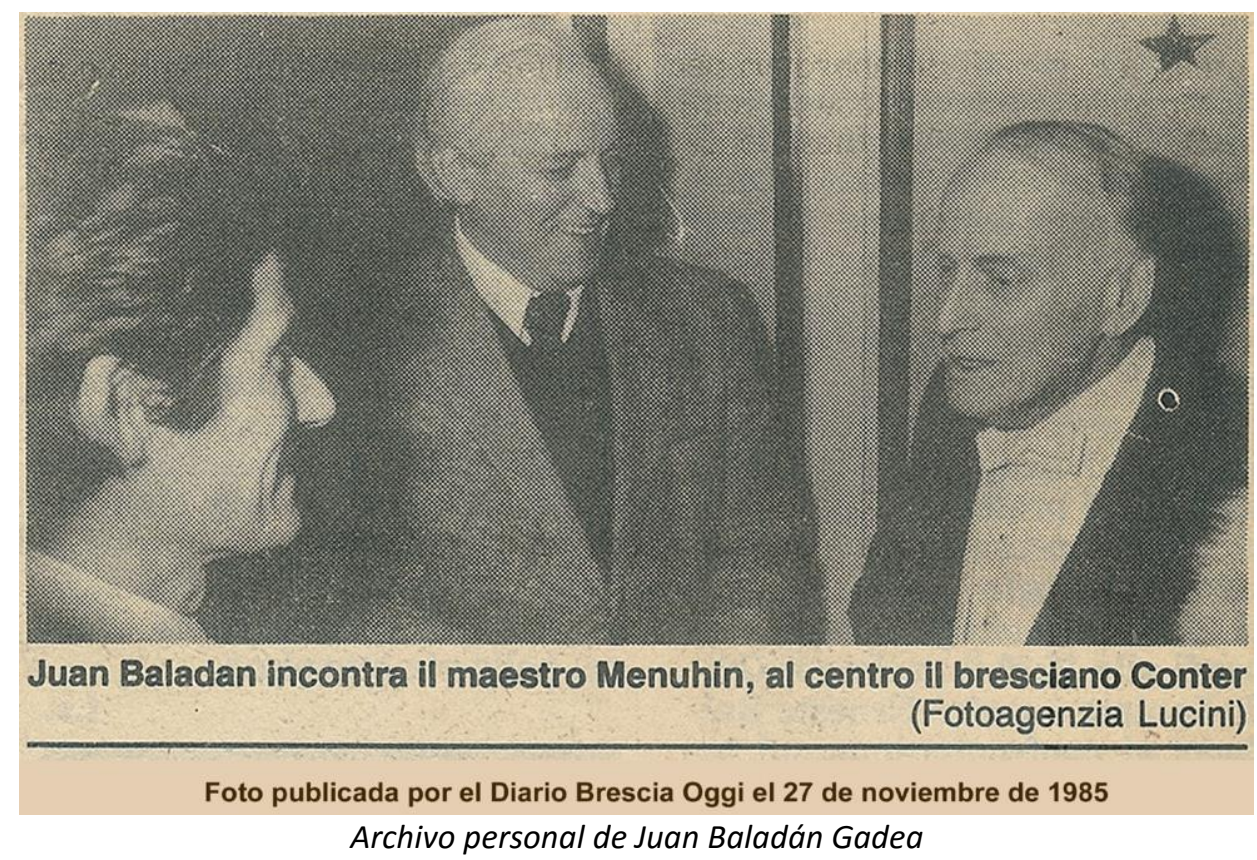

M. C. Cuándo lo liberaron en 1985, ¿se exilió directamente en Italia? ¿Por qué eligió ese país?

J. B. G. Llegué a Italia el 5 de abril de 1985, es decir, poco menos de un mes después de mi liberación. En un primer momento no elegí este país, vine a Italia porque mi esposa y mi hija se habían refugiado aquí en 1981. Después del referendum, que los militares perdieron ruinosamente, mi esposa comenzó a recibir llamadas telefónicas, tarde en la noche y a veces en la madrugada, en las cuales una voz anónima la amenazaba y terminaba por decirle que se fuera del país. Ella pensó en exiliarse -aunque con mucha incertidumbre- pero las dificultades que encontraba para trabajar siendo la esposa de un prisionero político y pensando que en el exterior podría encontrar mejores condiciones de vida, para ella y mi hija, la estimularon a que tomara la decisión de emprender el camino del exilio. Durante los meses de mayo y junio de 1985 hice una gira por varios países europeos: 
España, Francia, Holanda, Dinamarca, Suecia, Alemania y Suiza. Había muchas posibilidades de trabajo en el campo de la música; pero decidí volver a Italia y construírme un futuro con mi familia: mi esposa y mi hija me habían esperado por trece años y medio.

M. C. ¿Cuándo volvió por primera vez a Uruguay? ¿Vuelve regularmente? ¿Alimenta vínculos profesionales o personales en ese país? ¿Continúa su vida profesional?

J. B. G. Volví por primera vez a Uruguay en 1995, habían pasado diez años desde mi liberación, tiempo que me sirvió para reflexionar sobre la experiencia vivida y al mismo tiempo para integrarme en la sociedad de este país que me acogió generosamente. Cada tanto vuelvo a Uruguay, paso la mayor parte del tiempo en mi ciudad, Treinta y Tres, donde viven mis viejos amigos con los cuales he compartido tantos sueños (algunos los hemos realizado) y también han surgido nuevas amistades con personas más jovenes, que me ponen al paso de un tiempo nuevo. Cuando estoy en mi ciudad realizo muchos paseos por la campaña cercana o me voy al río Olimar a meditar. En el exilio he recibido muchas cosas que han hecho mi vida más rica; pero me falta siempre el trato sencillo de la gente y la belleza del tiempo lento de mi pago. Desde que llegué a Europa he mantenido relación constante con mi familia, amigos, músicos y escritores. Hasta los primeros años noventa a través de cartas y por teléfono, que poco a poco sustituí con los nuevos medios de comunicación, aunque si bien al teléfono lo utilizo mucho todavía. Continúo dedicándome a la poesía y a la composición, es decir continúo mi vida normalmente. 